\title{
CARACTERISTICAS EPIDEMIOLOGICAS DE 105 CASOS DE CRIPTOCOCOSIS DIAGNOSTICADOS EN LA REPÚBLICA ARGENTINA ENTRE 1981-1990.
}

\author{
A.J. BAVA \& R. NEGRONI
}

\section{RESUMEN}

Se presentan 105 casos de criptococosis diagnosticados en la República Argentina, entre 1981 y 1990 . El número anual de casos fue hasta 1987, de 4 a 8 , aumentó desde 1988 por influencia del SIDA y llegó a 35 casos en 1990. La criptococosis no asociada al SIDA se mantuvo entre 3 y 7 casos anuales.

Globalmente, el grupo etario más afectado fue el de 20-39 años y la distribución por edad fue diferente en las poblaciones con y sin SIDA. La mediana de la edad de las poblaciones total, asociada al SIDA y a otras causas predisponentes fue de 30,30 y 45 años, respectivamente. El predominio del sexo masculino fue mucho más evidente entre los pacientes $\mathrm{HIV}^{+}$que en los que no padecían esta última infección. La causa predisponente fue el SIDA en 57 pacientes, otra en 20 y era desconocida en 28 casos. Estimando indirectamente, el porcentaje de pacientes con SIDA que padecieron criptococosis en este período fue 6,19\% (57 casos en $920 \mathrm{HIV}^{+}$).

Coyplococcus neoformans variedad neoformans fue aislado de 101 pacientes y la variedad gattii (serotipo B) de los 4 restantes. Los datos obtenidos son similares a los observados en Europa y Estados Unidos.

UNITERMOS: Criptococosis; Cryptococcus neoformans; SIDA.

\section{INTRODUCCION}

La criptococosis es una micosis sistémica producida por un hongo levaduriforme capsulado: el Cryptococcus neoformans. Provoca severos cuadros infecciosos en huéspedes portadores de fallas de la inmunidad mediada por células. Se destacan entre sus causas predisponentes los trasplantes de órganos ${ }^{19}$, las enfermedades linfoproliferativas ${ }^{4}$, los tratamientos con drogas antiblásticas y corticoides ${ }^{12}$ y fundamentalmente el SIDA $A^{5,7,11,13}$.

Existen 2 variedades de Cryptococcus neoformans: Cryptococcus neoformans variedad neoformans (serotipos A y D) y Cryptococcus neoformans variedad gattii (serotipos B y C) ${ }^{8,17}$. Desde el punto de vista epidemiológico, la variedad neoformans es cosmopolita y se aisla de excrementos de palomas. Produce la mayor parte de los casos de criptococosis en pacientes con SIDA en todo el mundo inclusive en aquellas áreas donde la variedad gattii es predominante $e^{8,14,18}$. El hábitat de la variedad gattii se ha relacionado en los últimos años con el árbol Eucaliptus camaldulensis ${ }^{7}$ y su recuperación de casos clínicos ocurre mayoritariamente en áreas tropicales y subtropicales, consideradas como endémicas ${ }^{8,14,17}$. La diferenciación en variedades puede hacerse medios de cultivo selectivos, que ponen de manifiesto propiedades bioquímicas o bien mediante la serotipificación ${ }^{8,15}$.

En la Argenina, la primera comunicación de criptococosis fue hecha por Flavio Niño, en 1929. Desde ese momento y hasta 1959, sólo se habían comunicado una treintena de $\operatorname{casos}^{15}$. En la actualidad no se cuenta, hasta donde nosotros pudimos averiguar, con cifras oficiales de incidencia de esta enfermedad.

El propósito de este trabajo es el estudio de algunas características epidemiológicas de

Centro de Micologia. Departamento de Microbiología. Facultad de Medicina. Universidad de Buenos Aires. Paraguay 2155. Piso 11. 1121. Buenos Aires. Argentina. 
BAVA, A.J. \& NEGRONI, R. - Caracteristicas epidemiologicas de 105 casos de criptococosis diagnosticados en la Republica Argentina entre 1981-1990. Rev. Inst. Metl. trop. S. Paulo, 34(4):335-340, 1992

la criptococosis en la República Argentina, incluyendo la variedad predominante, a partir de una muestra de 105 aislanientos de Ciyptococcus neoformans estudiados en el Ccntro de $\mathrm{Mi}$ cologia de la Universidad de Buenos Aires.

\section{MATERIALES Y METODOS}

Diagnóstico de Cryptococcus neoformans: se estudiaron 105 aislamientos de hongos levaduriformes realizados en diferentes centros asistenciales de la República Argentina y que fueron enviados para su estudio al Centro de Micología, durante el período 1981-1990.

Las cepas fueron tipificadas através de las pruebas de producción de ureasa en medio de Christensen, crecimiento a $37^{\circ} \mathrm{C}$, auxanogramas de nitrógeno y carbono, producción de fenoloxidasa en medio de agar semilla de girasol $^{1} \mathrm{e}$ inoculación al ratón blanco ${ }^{17}$.

Datos epidemiológicos: para cada uno de los casos comunicados se requirió fecha y lugar de diagnóstico, edad y sexo del paciente y la presencia o no de causas predisponentes.

Determinación de variedad: se utilizó el medio propuesto por SALKIN \& IUUD ${ }^{18}$ que conticne $1,6 \mu \mathrm{g} / \mathrm{ml}$ de cicloheximida que inhibe selectivamente a la variedad neoformans; glicina como única fuente de carbono (utilizada como tal sólo por la variedad gattii) y rojo fenol como indicador de $\mathrm{pH}$.

Las cepas sembradas en este medio se incubaron a $28^{\circ} \mathrm{C}$ durante 1 semana y fueron observadas para detectar el cambio o no de color del medio (del amarillo al rojo). Las cepas que viraron el color del indicador al rojo fueron identificadas como variedad gattii y enviadas al National Institute of Health para su serotipificación.

\section{RESULTADOS}

1. Número y causas predisponentes de los casos de criptococosis comunicados al Centro de Micología, entre 1981-1990.

En la Tabla 1 se observa el incremento anual del número de casos de criptococosis estudiados en el Centro de Micologia de la U.B.A. en el período 1981-1990. Este aumento se produce fundamentalmente a expensas de la aparición de un nuevo factor predisponente: la infección por HIV. A comienzos de la década del 80 no existián pacientes con criptococosis asociada a esta infección en nuestro país, en tanto que en 1990 más del $85 \%$ de los casos se presentaban asociados a ella.

Por el contrario, los casos de criptococosis asociados a otras causas predisponentes se mantuvieron constantes a lo largo del período 1981-1990.

De los casos estudiados, 57 (54\%) padecían de SIDA, 20 (19\%) tenían otra causa predisponente, en $12(11 \%)$ ésta no fue determinada no obstante su búsqueda y en $16(15 \%)$ ese dato no nos fue proporcionado. Las causas predisponentes diferentes de la infección por HIV, entre las que resaltan los tratamientos inmunodepresores, se observan en la Figura 1.

TABLA 1

Número y porcentaje anual de casos de criptococosis comunicados entre 1981-1990 al Centro de Micologia de la U.B.A.

\begin{tabular}{|c|c|c|c|c|c|c|c|}
\hline \multirow[b]{2}{*}{ Año } & \multirow[b]{2}{*}{ total } & \multicolumn{3}{|c|}{ casos de criptococosis } & \multirow[b]{2}{*}{ SIDA } & \multirow[b]{2}{*}{ SIN } & \multirow[b]{2}{*}{ datos } \\
\hline & & $\mathrm{CON}$ & SIDA & NO & & & \\
\hline 1981 & 5 & 0 & $(0)^{*}$ & 5 & $(100,00)$ & 0 & $(0)$ \\
\hline 1982 & 4 & 0 & $(0)$ & 2 & $(50,00)$ & 2 & $(50,00)$ \\
\hline 1983 & 5 & 2 & $(40,00)$ & 1 & $(20,00)$ & 2 & $(40,00)$ \\
\hline 1984 & 7 & 3 & $(42,85)$ & 3 & $(42,85)$ & 1 & $(14,28)$ \\
\hline 1985 & 7 & 0 & $(0)$ & 5 & $(71,42)$ & 2 & $(28,57)$ \\
\hline 1986 & 8 & 4 & $(50,00)$ & 3 & $(37,50)$ & 1 & $(12,50)$ \\
\hline 1987 & 6 & 2 & $(33,33)$ & 2 & $(33,33)$ & 2 & $(33,33)$ \\
\hline 1988 & 12 & 6 & $(50,00)$ & 3 & $(25,00)$ & 3 & $(25,00)$ \\
\hline 1989 & 16 & 10 & $(62,50)$ & 4 & $(25,00)$ & 2 & $(12,50)$ \\
\hline 1990 & 35 & 30 & $(85,71)$ & 4 & $(11,42)$ & 1 & $(2,85)$ \\
\hline COTAL & 105 & 57 & $(54,28)$ & 32 & $(30,47)$ & 16 & $(15,23)$ \\
\hline
\end{tabular}

* Entre paréntesis, porcentaje sobre el total de casos de criptococosis. 
BAVA, A.J. \& NEGRONI, R. - Caracteristicas epidemiologicas de 105 casos de criptococosis diagnosticados en la Republica Argentina entre 1981-1990. Rev. Inst. Med. trop. S. Paulo, 34(4):335-340, 1992

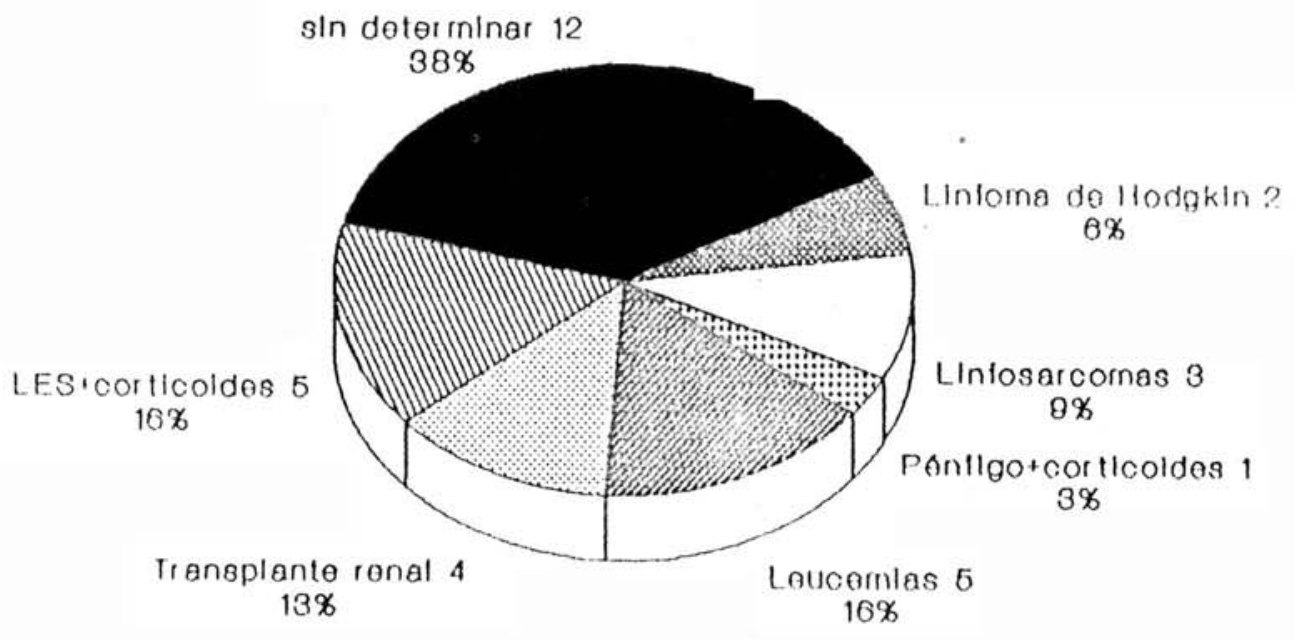

Número lotal de pacientes con causas predisponentes dilerentes del SIDA o blen no determinada no obstante su búsqueda $=32$

Fig. 1: Número y porcentaje de pacientes con criptococosis en los cuales se encontró una causa predisponente diferente del SIDA o bien ésta no pudo ser determinada no obstante su búsqueda, entre los casos comunicados al Centro de Micologia durante el período 1981-1990.

\section{Edad de los pacientes estudiados}

Sólo se obtuvo la edad en 64 casos $(61 \%)$ debido a que muchos aislamientos se enviaban con información incompleta. De ellos, 44 eran pacientes con criptococosis asociada al SIDA,

\section{TABLA 2}

Distribución por grupos etarios de 105 casos de criptococosis comunicados al Centro de Micología de la U.B.A., durante el período 1981-1990.

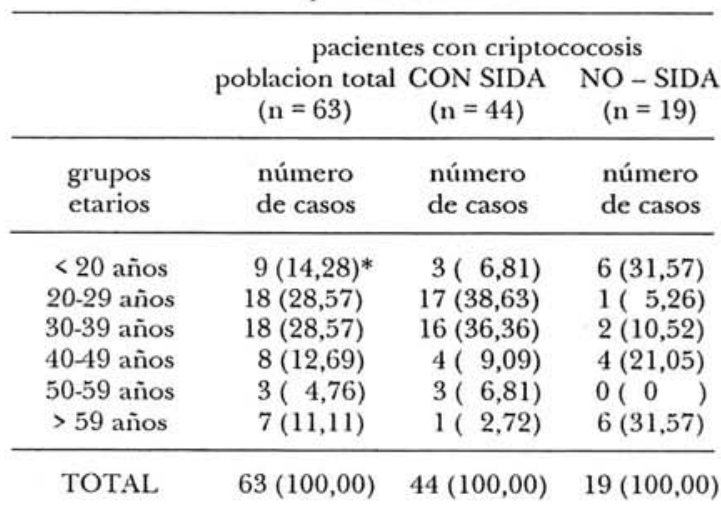

* Entre paréntesis, porcentaje sobre el total de casos de criptococosis.
19 a otras causas predisponentes y uno sin factor de riesgo conocido (de 21 años de edad).

Tal como se observa en la Tabla 2, los pacientes infectados con HIV pertencían en su mayoría $(75 \%)$ a las edades comprendidas entre los 20-39 años, en tanto que las edades de los $\mathrm{HIV}^{-}$se distribuían más heterogéneamente.

La mediana de la edad de la población estudiada fue de 30 años (rango: 6-71 años), de 40 años (rango: 6 - 71 años) en la población no SIDA y de 30 años (rango: 19-61 años) en los casos asociados a la infección por HIV.

\section{Distribución por sexos de la población es- tudiada}

El sexo fue conocido en 96 casos (91\%) y tal como se observa en la Figura 2 la distribución de los casos de criptococosis por sexos mostró un predominio masculino (aproximadamente 3:1) debido a la influencia de los pacientes $\mathrm{HIV}^{*}$. En este último grupo el predominio masculino fue más evidente $(>90 \%)$, mientras que en la población $\mathrm{HIV}^{-}$las diferencias entre ambos sexos fueron mucho menores ( $45 \%$ de hombres y $37 \%$ de mujeres). 
BAVA, A.J. \& NEGRONI, R. - Caracteristicas epidemiologicas de 105 casos de criptococosis diagnosticados en la Republica Argentina entre 1981-1990. Rev. Inst. Med. trop. S. Paulo, 34(4):335-340, 1992

4. Variedades de Cryptococcus neoformans recuperadas de los casos estudiados

De las 105 cepas de Cryptococcus neoformans estudiadas, sólo $4(3,80 \%)$ viraron el color del medio de glicina, ciclohemida y rojo fenol y fueron clasificadas como variedad gattii. Todas ellas fueron identificadas como serotipo B en el National Institute of Health. Las 101 cepas restantes $(96,19 \%)$ pertencieron a la variedad neoformans. Todos los casos de criptococosis asociada al SIDA fueron producidos por la variedad neoformans.

\section{Sitios de diagnóstico de los casos estu- diados}

Noventa ( $85 \%$ ) de los 105 casos estudiados provinieron de centros asistenciales ubicados en la ciudad de Buenos Aires, 4 (4\%) de centros ubicados en el Gran Buenos Aires y 11 (11\%) del interior del país. Si bien el sitio de diagnóstico no es obligatoriamente el de residencia del paciente, en la gran mayoría es el mismo e a lo sumo una ciudad próxima.

\section{CONCLUSIONES}

En este trabajo hemos presentado datos de 105 pacientes con criptococosis que confirman su creciente importancia como infección oportunista en huéspedes inmunocomprometidos, especialmente en los $\mathrm{HIV}^{+}$. Estos últimos constituyen el grupo de riesgo más importante y su frecuencia está en ascenso. En ellos la criptococosis adquiere caracteres particulares, convirtiéndose en la infección micótica marcadora de SIDA más severa y la segunda en frecuencia, detrás de la candidiasis esofágica $^{(5,7,11,13)}$. Entre el 6 y el $20 \%$ de los pacientes con SIDA enferman de criptococosis y la casi totalidad de ellos muere a causa de esta infección, no obstante la aplicación de una terapéutica específica ${ }^{(12)}$.

Asumiendo que hubiéramos estudiado la totalidad de los casos de criptococosis asociada al SIDA ocurridos en la República Argentina en el período 1981-1990, nuestros 57 pacientes representarían un $6,19 \%$ del total (920 casos de SIDA hasta 1990, ref. 6). Esta cifra, que es obviamente una subestimación, concuerda con

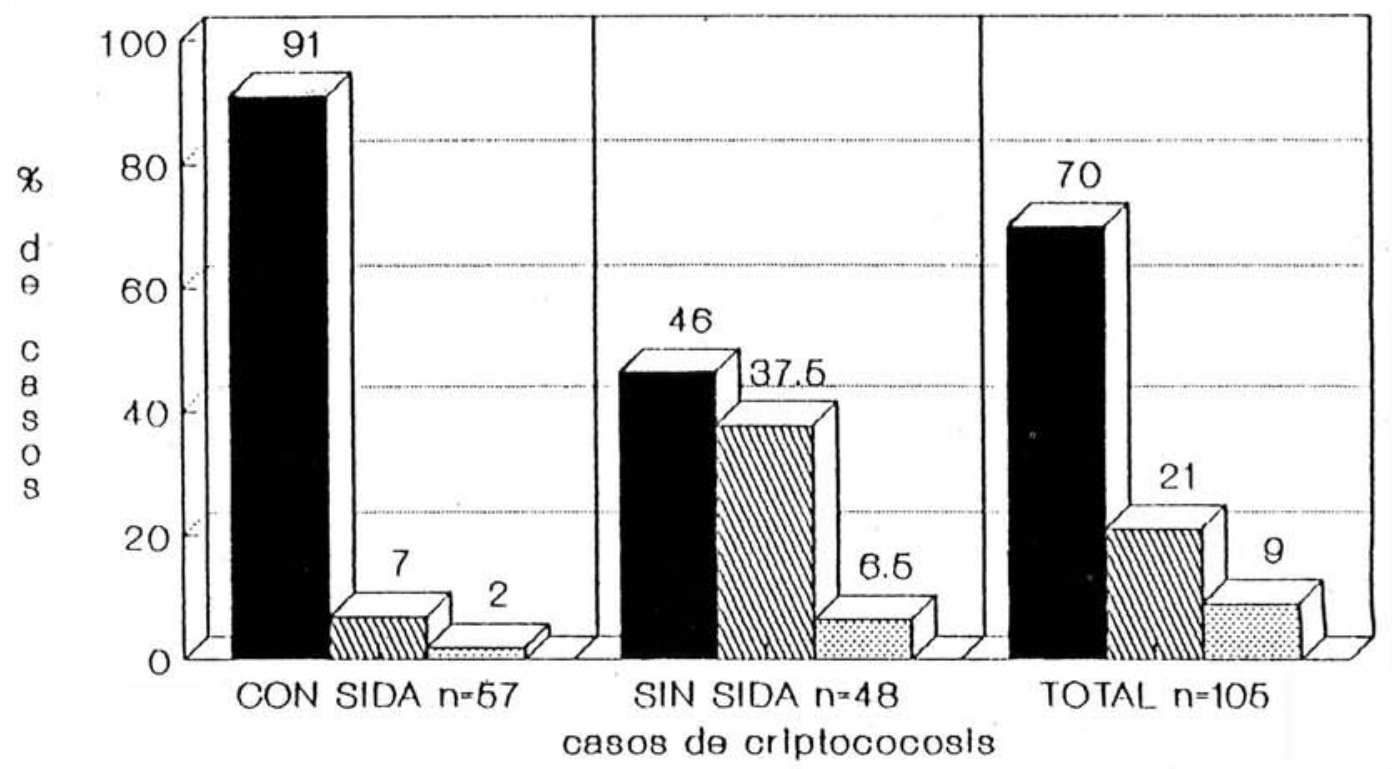

sexo masculino Allill sexo leminino dato desconocldo

Fig. 2: Predominio del sexo masculino observado en la distribución por sexos de 105 pacientes con criptococosis (asociada y no asociada al SIDA) comunicados al Centro de Micologia, durante el período 1981-1990. 
BAVA, A.J. \& NEGRONI, R. - Caracteristicas epidemiologicas de 105 casos de criptococosis diagnosticados en la Republica Argentina entre 1981-1990. Rev. Inst. Med. trop. S. Paulo, 34(4):335-340, 1992

las comunicadas en Estados Unidos, Brasil o el continente europeo ${ }^{(10,14,16)}$.

No obstante este incremento en el número total de casos, la criptococosis no asociada al SIDA ha permanecido con valores estables durante el período estudiado. Otras causas predisponentes han sido aquellas habitualmente reconocidas como tales: tratamientos inmunodepresores, leucemias, linfomas, transplantes de órganos, $\operatorname{ctc}^{(4,8,10,17)}$.

En nuestra casuística podimos detectar una causa predisponente en el $73 \%$ de los casos. Esta cifra es similar a la comunicada en Estados Unidos y Europa (70-85\%) y superior al $30 \%$ descripto en $\mathrm{Asia}^{(10)}$.

La prevalencia de la variedad neoformans como agente etiológico de la criptococosis en esta muestra confirma los datos obtenidos en un estudio anterior ${ }^{(2)}$ y su recuperación de todos los casos asociados al SIDA es coincidente con lo ocurrido en el resto del mundo ${ }^{(8,1-4,15,17)}$. En el Centro de Micología hemos tomado conocimiento hasta la fecha, en el Centro de Micología, de 4 casos de criptococosis producida por la variedad gattii, todos en individuos HIV-. Uno de ellos nunca había salido de los límites del país lo que nos hace pensar que este hongo puede encontrarse en determinadas áreas tropicales o subtropicales de la Argentina, donde además se encuentra ampliamente distribuido el árbol Eucaliptus camaldulensis ${ }^{(7)}$.

La distribución por sexos de la población estudiada mostró un $70 \%$ de casos en varones lo cual es coincidente con la bibliografía ${ }^{(8,20)}$. Sin embargo al comparar las poblaciones con y sin SIDA como causa predisponente, se observa que el predominio masculino sólo es evidente en el primer grupo.

La mayor parte de los casos incluidos en este estudio provinieron de centros asistenciales ubicados en la ciudad de Buenos Aires y sus alrededores, donde la concentración poblacional y las posibilidades de diagnóstico son mayores. La falta de un número más elevado de casos procedentes del interior del país se debe probablemente a subregistro y a dificultades de comunicación entre los centros asistenciales allí ubicados y el Centro de Micología.

La criptococosis no presenta en nuestro medio características que la distingan de la observada en el resto del mundo. Hoy vemos 2 tipos diferentes de criptococosis: una relativamente nueva, asociada al SIDA, actualmente mayoritaria y otra, clásica, asociada a otras causas predisponentes, que no ha variado en sus caracteres clínicos y epidemiológicos a traves de los años.

\section{SUMMARY}

\section{Epidemiological characteristics of 105 cases of Cryptococcosis diagnosed in Argentina, between 1981-1990}

Some epidemiological characteristics of 105 cases of cryptococcosis diagnosed in Argentina, between 1981-1990, were studied. Until 1987, the annual number of cases was 4-8. It has increased since 1988 by AIDS influence and reached 35 cases in 1990. The annual number of cases non associated with AIDS has remmained in 3-7 cases.

The age median of the all patients, AIDS and non AIDS associated cases was 30, 30 and 45 years old, respectively. The $20-39$ years old group was the most affected and the age distribution was different in AIDS and non AIDS population.

Masculine predominance was more evident in AIDS than in non AIDS associated cases.

The predisposing factor was AIDS in 57 patients, another different factor in 20 and unknown in 28 cases. The indirect estimation of the percentage of AIDS cases with cryptococcosis was $\geq 6,19 \%\left(57\right.$ cases in $\left.920 \mathrm{HIV}^{+}\right)$during 1981-1990 this period.

Ciyptococcus neoformans variety neoformans was isolated from 101 cases and the gattii variety (serotype B) from only 4.

The obtained data are similar to those observed in Europe and United States.

\section{AGRADECIMIENTOS}

A la Dra. K.J.Kwon Chung, del National Institute of Health, por la serotipificación de las cepas de Cryptococcus neoformans var. gattii. A los Dres. O. Libonati y M.Calello del Centro de Referencia de SIDA de la Facultad de Medicina, por la entrega de datos acerca del SIDA y al Dr. R. A. Diez por su ayuda en la corrección de este trabajo. 
BAVA, A.J. \& NEGRONI, R. - Caracteristicas epidemiologicas de 105 casos de criptococosis diagnosticados en la Republica Argentina entre 1981-1990. Rev. Inst. Med. trop. S. Paulo, 34(4):335-340, 1992

\section{REFERENCIAS BIBLIOGRÁFICAS}

1. BAVA, A.J. \& NEGRONI, R. - Estudio epidemiológico sobre criptococosis en San Pedro (Buenos Aires). Rev. argent. Micol., 9(3):12-16, 1986.

2. BAVA, A.J. - Diferenciación en pares serotípicos de cepas de Cryptococcus neoformans aisladas en la Argentina. Rev. argent. Micol., 10(2):12-13, 1987.

3. BAVA, A.J. \&c NEGRONI, R. - Estudio del primer caso autóctono de la Argentina de criptococosis por Cryptococcus neoformans var. gattii. Rev. iberoamer. Micol., $7: 39-42,1990$.

4. COLLINS, V.P.; GELLHORN, A. \& TRIMBLE, J.R. The coincidence of cryptococcosis and disease of the reticuloendothelial and lymphatic systems. Cancer, 4:883-889, 1979.

5. CHUCK, S.I. \& SANDE, M.D. - Infections with Cryptococcus neoformans in the acquired immunodeficiency syndrome. New Engl. J. Med., 321:794-799, 1989.

6. DEPARTAMENTO DE SIDA y E.T.S. Secretaría de Salud. Ministerio de Salud y Acción Social de la República Argentina. Boletín Informativo: Notificación de casos de SIDA en la República Argentina. [Mimeo de Ministerio de Salud y Acción Social, 1992.]

7. DE VITA, V.T.; HELLMAN, S. \& ROSEMBERG, S.A. - SIDA, Etiología, diagnóstico, tratamiento y prevención. Barcelona, Salvat Editores, 1986. p. 161-184.

8. DIAMOND, R.D. - Cryptococcus neoformans. In: MANDELL, G.L.; DOUGLAS, R.C. \& BENNETT, J.E. ed. Principles and practice of Infectious Diseases. New York, John Willey, 1987. p. 1460-1468.

9. ELLIS, D.H. \& PFEIFFER, T.J. - Natural habitat of Cryptococcus neoformans var. gattii. J. clin. Microbiol., 28:1642-1644, 1990.

10. DROUHET, G. \& DUPONT, B. - Cryptococcosis. In: JACOBS, P. H. \& NALL, L. - Antifungal drugs therapy. New York, Marcel Dekker, 1990. p. 143-164.
11. HAY, R. J. - Clinical manifestations and management of cryptococcosis in the compromised patient. In: WARNOCK, D. W. \& RICHARDSON, M.D. - Fungal infection in the compromised patient. New York, John Wiley \& Sons, 1982. Chapter 4, p. 93-117.

12. KHAN, M.A. \& SBAR, S. - Cryptococcal meningitis in steroid-treated systemic lupus erythematosus. Postgrad. med. J., 51:660-664, 1975.

13. KOVACS, J.A.; KOVACS, A.A.; POLIS, M.; WRIGHT, W.C.; GILI, V.J.; TUAZON, V.C.; GELMANN, E.P.; LANE, H.C.; LONGFIELD, R.; OVERTURF, G.; MACHER, A.M.; FAUCI, A.S.; PARILLO, J.E.; BENNETT, J.E. \& MASUR, H. - Cryptococcosis in the acquired immunodeficiency syndrome. Ann. intern. Med., 103:533-538, 1985.

14. NEGRONI, R. - Micosis en pacientes con SIDA. Rev. argent. Micol., 13 (1): 3-14, 1990.

15. NIÑO, F.L. - Criptococosis. In: Lecciones de Micología y Micopatología. Buenos Aires, Editorial Cajica, 1959. cap 13, p. 275-292.

16. PATTERSON, T.F. \& ANDRIOLE, V.T. - Current Concepts in Cryptococcosis. Europ. J. clin. Microbiol. infect. Dis., 8:457-465, 1990.

17. RIPPON, J.W. - Medical Mycology: the pathogenic Fungi and the pathogenic Actinomycetes. 3. ed. Philadelphia, W.B. Saunders, 1988. p. 582-609.

18. SALKIN, I.F. \& HURD, N.J. - New media for differentiation of Cryptococcus neoformans serotypes pairs. J. clin. Microbiol., 15, 169-171, 1982.

19. SCHOROTER, G.P.J.; TEMPLE, D.R.; HUSBERG, B.S.; WEIL, R.W. \& STARZL, T.E. - Cryptococcosis after renal transplantation. Report of ten cases. Surgery, 79:268-277, 1976.

20. TRESERLER, C.B. \& SUGAR A.M. - Fungal Meningitis. Infect. Dis. Clin. N. Amer. 4:789-808, 1990.

Recebido para publicação em 19/04/1991 Aceito para publicação em 15/06/1992 